\title{
First report of lettuce big-vein associated varicosavirus infecting lettuce in China
}

\author{
Guo-Hua Zheng ${ }^{1}$ (I) $\cdot$ Ting Ye ${ }^{2} \cdot$ Fu-Rong Liao ${ }^{3} \cdot$ Wen-Zhu Zhang ${ }^{1} \cdot$ Yan-Lin Ming ${ }^{1}$ \\ Received: 8 October 2018 / Accepted: 20 November 2018 / Published online: 23 January 2019 \\ (C) Società Italiana di Patologia Vegetale (S.I.Pa.V.) 2019
}

Keywords Stunted growth $\cdot$ RT-PCR $\cdot$ Genomic sequence $\cdot$ Phylogenetic analysis

In December 2016, four lettuce plants exhibiting stunting were collected from a farm in Xiamen city, Fujian province, China. RT-PCR was employed for detection of lettuce bigvein associated varicosavirus ( $\mathrm{LBVaV})$, Mirafiori lettuce big vein virus, lettuce necrotic yellows virus, cucumber mosaic virus, potyviruses and tobamoviruses. One sample (xm) was found infected with LBVaV by nested RT-PCR using primers LBV-F1 (5'-CTCCACGCTCAAGATGTTCGC-3'), LBV-R1 (5'-CGCCACCTGTCTCGTGTACC-3'), LBV-F2 (5'CTGGATGGACTCAGGCTTGG-3') and LBV-R2 (5'CAAGATGGCAACGGTATCTGC-3'), according to a previous report (Matsumoto et al. 2004). Virions were semipurified from leaves $(20 \mathrm{~g})$ by two differential centrifugations, as described by Kuwata et al. (1983). The RNA of the semipurified virions was extracted using a TransZol Plant kit (Transgen Biotech Co., Ltd., China) and used to prepare a RNA-seq library using VAHTS ${ }^{\mathrm{TM}}$ mRNA-seq V2 Library

Yan-Lin Ming

xmyanlin@gmail.com

1 Key Laboratory of Xiamen City for Plant Introduct \& Quarantine and Plant Product, National Plant Introduction \& Quarantine Base, Xiamen Overseas Chinese Subtropical Plant Introduction Garden, Xiamen 361002, China

2 Fujian Institute of Subtropical Botany, Xiamen 361006, China

3 Inspection and Quarantine Technology Center, Xiamen Entry-Exit Inspection and Quarantine Bureau, Xiamen 361026, China
Prep Kit for Illumina ${ }^{\circledR}$ (Vazyme Biotech Co., Ltd., China), and sequenced in 150-bp paired-end HiSeq2500 sequencing reactions at the Sangon Biotech Co., Ltd. (China). A total of 942,848 reads was obtained. The near full-length sequence of RNA1 (6639 nt, MH349091) and RNA2 (5999 nt, MF045444) were determined. The sequences showed 95.298.1\% (RNA1) and 94.7-99.4\% (RNA2) nucleotide sequence identities with other LBVaV isolates, and were closest to Japanese isolates (AB114138 and AB075039 with $98.1 \%$ and $99.4 \%$, respectively). Phylogenetic analysis confirmed that LBVaV isolate $\mathrm{xm}$ is closely related to isolates from Japan. To our knowledge, this is the first report of LBVaV in China.

Acknowledgements This work is supported by Xiamen Science and Technology Bureau (3502Z20162013, 3502Z20162014 and 3502Z20161239). Guo-Hua Zheng and Ting Ye contributed equally to this work.

Publisher's Note Springer Nature remains neutral with regard to jurisdictional claims in published maps and institutional affiliations.

\section{References}

Kuwata S, Kubo S, Yamashita S, Doi Y (1983) Rod-shaped particles, a probable entity of lettuce big vein virus. Jpn J Phytopathol 49(2): 246-251

Matsumoto J, Tamaki K, Shiwaku K, Sasaya T, Ishikawa K (2004) Detection of lettuce big-vein virus and mirafiori lettuce virus by reverse transcription-polymerase chain reaction. Bull Hyogo Pre Tech Cent Agr Forest Fish (Agriculture) 52:1-6 